\title{
Happier Together: Integrating a Wellness Application Into a Social Network Site
}

\author{
Sean A. Munson, Debra Lauterbach, Mark W. Newman, and Paul Resnick \\ School of Information, University of Michigan \\ 1075 Beal Ave, Ann Arbor, MI 48109 \\ \{samunson, dlauter, mwnewman, presnick\}@umich.edu
}

\begin{abstract}
What are the benefits and drawbacks of integrating health and wellness interventions into existing online social network websites? In this paper, we report on a case study of deploying the Three Good Things positive psychology exercise as a Facebook application. Our experience shows that embedding a wellness intervention in an existing social website is a viable option. In particular, we find adherence rates on par with or better than many other Internet-based wellness interventions. We also gained insights about users' privacy and audience concerns that inform the design of social networkbased wellness applications. Participants did not want all of their entries to be shared with all their Facebook friends, both because they did not want others to know some things and because they did not want to clutter others' newsfeeds. Users found it compelling, however, to interact with their friends around some "Good Things" they had posted.
\end{abstract}

Keywords: Social software, wellness, positive psychology, design, privacy, sharing, audience

\section{Introduction}

Applications to promote health and wellness have attracted significant attention from the HCI and Health Informatics research communities [e.g., 1-10]. Increasingly, applications allow intervention participants to interact with each other [e.g., 3, 4, 7]. Typically, however, they are deployed as stand-alone sites. We explore the benefits and design challenges of integrating a wellness application into an existing online community with a large and active membership that was not developed explicitly to support health interventions. In particular, we built and deployed a Facebook application called "3GT" that supports the Three Good Things exercise developed by positive psychologists Seligman et al. [11] to promote psychological well being.

Building health and wellness applications into a popular networking site like Facebook appears promising for several reasons. First, the application can draw on participants' existing social networks rather than asking them to form new relationships with other participants. For certain types of supportive exchanges, participants may get more benefit from interacting with people with whom they already share a bond, especially in the near term $[12,13]$. Second, many members of 
Facebook visit the site very frequently, with nearly $50 \%$ of active users visiting every day [14]. Integrating a health intervention into a website that people are already frequenting could help overcome the challenges to adherence that many self-directed health interventions face $[1,6,10]$. Reported adherence to Internet-mediated anxiety and depression interventions deployed through open-access stand-alone websites were as low as $1 \%$ [15]; participation rates in controlled studies, presumably involving subject screening and participation incentives, were reported as $19 \%$ for a smoking cessation study and $34 \%$ for a diabetes self-management study [16].

However, integrating with a social networking site like Facebook may also have drawbacks. First, data entry requires an Internet connection, which may be inconvenient for recording activities and thoughts at certain times. Second, the average list of Facebook contacts includes 120 people [14] and represents a variety of types of relationships, including both strong and weak ties [17]. Some information may be too intimate or too mundane to share with such a large and diverse audience.

In this project, we set out to gain a deeper understanding of the advantages and disadvantages of deploying a health intervention within Facebook. We were specifically interested in answering the following questions: Does sharing and interacting with existing friends affect user retention? Are people comfortable sharing all their activity from the wellness application with all their existing social network friends? If not, then what features should be offered for controlling the visibility of activity in a wellness application? Does building structured features tailored to a specific wellness activity offer benefits over repurposing generic features of the Facebook platform?

\subsection{Motivation and Background}

There have been many recent studies of online or computer-supported intervention to promote wellness - especially physical activity promotion [e.g., 1, 3, 6, 7, 9, 18], diet [e.g., 4], or chronic disease management [e.g., 8, 10]. Internet-based support groups for both patients and caregivers have also received substantial research attention [e.g., $2,5,19,20]$. These online support groups offer participants a place for social support and sharing of knowledge.

In between these two spaces, however, is a relatively unexplored space of integrating the social experience with the intervention. In addition to offering support and advice in the form of discussions, the persuasive power of group dynamics and social influence may make social software a particularly potent technology for promotion of behavior change [21]. Participants' goals and progress towards goals might be shared with other users of the application, or with their friends, depending on the context and type of intervention. A more public intervention, with progress recorded and shared, may make people feel more accountable and lead to better adherence to recommendations. Reminders or "nagging" to participate might come from friends rather than from the system [13].

Some previous studies of computer-supported wellness interventions have connected their participants [e.g. 4, 7, 18]. For example, Consolvo et al made participants' step counts visible to other participants in a physical activity study. They found that this sharing influenced participants' motivation through both social 
pressure and social support [18]. Integrating the behavior tracking with social interactions was a strength of the application. The Fish'n Steps application [7] placed people in teams in an intervention to increase their physical activity. The researchers found this generated both cooperation and competition. Team membership, however, did not increase motivation; participants generally felt awkward contacting anonymous team members, but participants who knew each other in person were eager to discuss the game and share progress in face-to-face interactions. Like previous research, our research examines the role of social sharing in a wellness intervention. Unlike previous work, we study the integration of a wellness intervention with social software that participants already use (Facebook) and share their activity in the application with their existing contacts (their Facebook social network) rather than with other intervention participants who they may not know in real life.

Psychological Wellness. Positive psychology - the "study of the strengths and virtues that enable individuals and communities to thrive" [11] - is an emerging research area. Its focus on helping people thrive contrasts with psychology's more prominent focus on treating mental illness. Positive psychologists have developed a number of exercises that help people live happier and more fulfilling lives [22]. Participants in one of the most effective exercises, Three Good Things, are supposed to record three good things each day and the reasons why these things happened. By focusing on the good, rather than dwelling on the negative, it is theorized that people can train themselves to be happier. Previous studies of this exercise [e.g., 22] have focused on offline, private participation: subjects received instructions online, but recorded their daily good things offline. Results have been consistent with the theory: subjects reported decreased symptoms of depression and increased happiness as compared to a baseline before the exercise.

In the wild, people have already adapted Three Good Things for the social websites they already use. People share their good things on Facebook groups, in public blogs, and a "dare" on the Livestrong site. Before developing and deploying our own application, we studied two existing Facebook groups dedicated to this intervention, one with 68 members and the other with 144 members as of August 2009. Both follow the same format, with group members posting good things to the group's wall, usually as a list of all three items at a time. The posts and comments on the Facebook groups' walls contain interaction between members and both social support and social pressure. Group members occasionally congratulate one another on a good thing, comment on them, or repeat another's good thing as one of their own. In one of the groups, one member in particular greeted all new members by name and encouraged them to post. This member also prompted existing members to post ("what about you guys? how'd your day go?"). In other cases, people shared if they were having a bad day and were unable to come up with three good things, and in one example, another group member actually came up with three things for someone having a bad day. Finally, some members reflected on how the exercise was making them feel: "Wow. It actually lifts my spirits to see people, I don't even know, joining this group and reflecting on even -a few- good things. It's nice to just share in all of that with you." 


\section{Three Good Things Application}

Based in part on insights from observing the Facebook groups, we built an application that could support the Three Good Things intervention, which we call 3GT (apps.facebook.com/threegoodthings). We included features that supported the key elements of the intervention as described in positive psychology literature, features that would support the social sharing we had observed in the Facebook groups, and the strengths identified in previous studies of integrating social experiences with computer-supported wellness interventions. We summarize the key features below.

Support for private and public recording of good things. Each good thing could be posted with one of three privacy options: shared on the participant's newsfeed (visible to all of their friends, Figure 1) and visible to their Facebook friends who visit their 3GT profile, visible to their Facebook friends who visit their 3GT profile, and private (visible only to the participant).

Structured support for good things and reasons. The theoretical literature on the exercise emphasizes the importance of reflecting on why good things happen. We did not see evidence that this was occurring in the posts to the Facebook groups. Our application allowed participants to list both a good thing that happened, and, in a separate and private field, the reason (see Figure 2). We hoped that explicitly asking for the reason in a separate field would encourage people to reflect on why the good things had happened to them.

Social support \& social pressure. By allowing participants to post Good Things to their Facebook newsfeed, and by making their 3GT profiles visible to their friends even if the friends were not 3GT users, we hoped to enable social support from friends, in the form of structured support in Facebook (e.g. "liking" someone's good thing or commenting on it) as well as unstructured interactions between individuals through other communication channels. By making participants' activity in the application visible to their friends, we believed users might remind friends to participate if they noticed they had not posted in a while. We also created a formal mechanism, user-to-user notifications. When viewing the profile of a friend who had not posted in more than two days, a participant could remind the friend to participate. Clicking would send a Facebook notification to the friend (Figure 3 right).

Integration with participants' routines and habits. In addition to being able to post from the 3GT application, participants were able to create public posts from outside

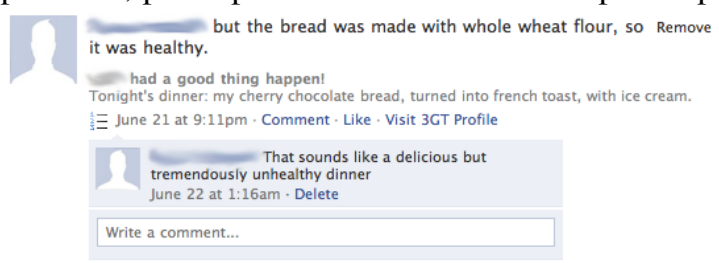

Fig. 1. Example of a Good Thing shared on a news feed or profile. Includes a comment from a Facebook friend of the participant. 


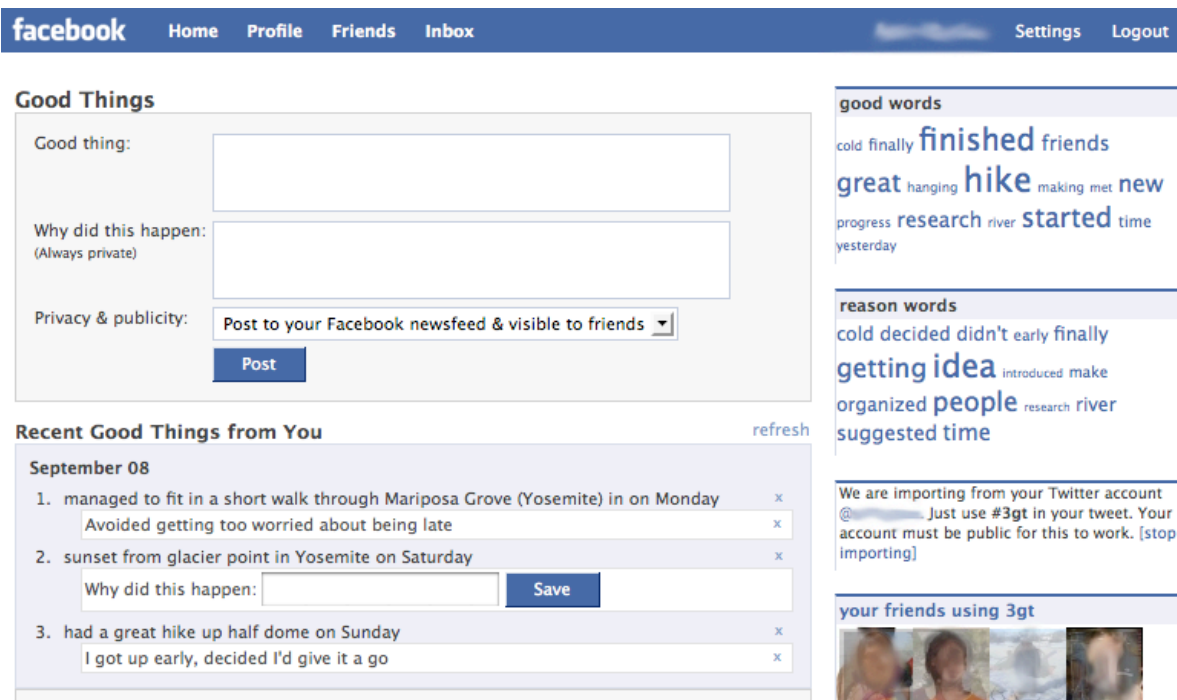

Fig. 2. Screenshot of the main 3GT application page. Left column: form for posting and a list of the participant's good things and reasons. Right column: word clouds, links to the participant's friends' 3GT profiles, and a link to an integrated timeline of all of the public good things from friends who are using $3 \mathrm{GT}$.

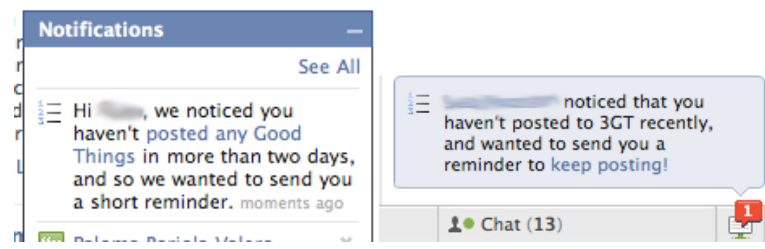

Fig. 3. Reminders sent through Facebook notification interface. Left: Example systemgenerated notification. Right: Example user-to-user notification.

of the 3GT application, by posting on their Facebook profile or newsfeed pages or by importing tweets containing the hashtag \#3gt from their Twitter account - both places where many people already post events that could be considered good things. We hoped that these additional integration points would increase adherence by reducing the steps required to post a good thing and by fitting into activities that participants already do. Some participants would also receive computer-generated reminders if they did not post for two days, and again after four days since their last post. These reminders would appear alongside other Facebook notifications when the participant logged into Facebook (Figure 3 left), likely an opportune time to post because they were already logged in.

\section{Study Overview}

3GT was publicly available as an application on Facebook starting on 20 July 2009. Participants consented to being in a research study and completed a questionnaire 
after installing the application. By 21 February 2010, 190 participants had completed the initial signup process for $3 \mathrm{GT}$. We recruited participants primarily through an advertisement we ran on Facebook (71 participants). Additional participants were recruited either after an invitation from a friend or seeing 3GT on a friend's profile (57 participants), finding 3GT in the Facebook application directory (24 participants), or reading about 3GT elsewhere on the Internet (38 participants). 20 additional participants who are Facebook friends of the researchers were excluded from our analysis. Participants were not compensated. The participants included 25 men (age mean 35.6 years, stdev 14.8 years) and 164 women (age mean 36.7 years, stdev 13.3 years); 1 participant did not specify gender.

We recorded participants' interactions with the 3GT application. We also completed semi-structured interviews with six participants. This included three participants who knew one or more of the researchers (though they were interviewed by a researcher who did not know them) and one participant who was a friend-of-afriend of one of the researchers.

In addition to our general goal of observing how users interacted with 3GT and what their perceptions of it were, we explored variations of the design to determine if they had any effects on usage. We deployed variants of the application along two dimensions: system-generated reminders to post good things and the default privacy or publicity of each good thing posted. Participants were assigned to one of four conditions randomly in a $2 \times 2$ design.

In the reminders condition, participants received reminders after 2 and 4 days without posts. Reminders were sent as Facebook notifications, which appear as subtle pop-ups when the user is logged in to Facebook (Figure 3 left). In the no-reminders condition, participants received such reminders only if their friends sent them.

In the private by default condition, good things added from the 3GT application page (Figure 2) were visible only to the participant. In the public default, good things were posted to the participants' walls, and thus had a chance of showing up in all their friends' news feeds. Participants could change the privacy on a per-post basis at the time of posting but could not change their defaults.

\section{Results}

Of the 190 participants who signed up for 3GT, who were not Facebook friends of the developers, and completed the pretest, many posted a few good things on their first visit and never returned. We refer to those as dropouts and restrict our analysis of usage to 55 participants who posted for at least one week, who we refer to as the active users. These participants were 36.9 years old, on average, and included 7 men (13\%) and 48 women (87\%). They were more female than participants in Seligman et al's study of offline positive psychology interventions (42\% male and 58\% female) [22]. We present usage data as of 1 March 2010, for active users who joined prior to 21 February 2010.

On average, active users posted 0.83 good things per day (Figure 4 ), defined as the total number of good things posted divided by the number of days between their first and last good thing posted. Though far less than the recommended level of 

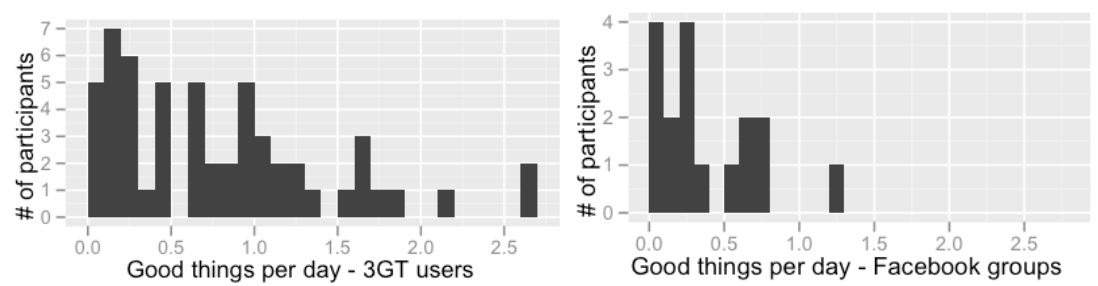

Fig. 4. Daily good things for active 3GT application users (left) vs. participants in the $3 \mathrm{GT}$ groups (right).

participation, this was quite a bit higher than the active participants (defined by the same criteria of posting for at least one week) in the Facebook groups, where active participants averaged 0.38 and 0.47 good things per day.

3GT users posted reasons for $80 \%$ of their good things, compared to $0 \%$ for the groups. This was true even when participants were unsure of the benefits of recording the reason. As one participant said, "I don't know what I'm supposed to write there, but there's a box there with a big blue button that says 'Save,' so I feel like I haven't finished it properly unless I write something. I feel a little bit like I have to finish it."

Of the good things posted by active users, $91 \%$ were posted from the application, $6 \%$ were posted from participants' newsfeeds or Facebook profiles, and $4 \%$ were imported from Twitter. Even though participants did not regularly use the most integrated form of posting (posting from their newsfeed or profile), the participants we interviewed were generally in agreement that having the application on Facebook was convenient, and that logging into Facebook would sometimes remind them to post. Though posting from Twitter was rare, participants felt that it had some particular strengths. One participant we interviewed reported "And I love that it imports things from Twitter, because I use that as well. So if I don't feel like putting it in Facebook I can put it in anyway. So having lots of ways to get things in there... less barrier to entry." Another participant commented on how easy it was to turn a tweet into a 3GT post by adding the hashtag as "an afterthought," and then go back fill in the reason the next time she visited the application. Another participant felt that posting in-the-moment from Twitter and later visiting the application to fill in reasons caused her to reflect more than if she had simply posted both at the same time.

\subsection{Privacy and Audience}

Of the 55 active participants, 28 were in the public default condition and 27 were in the private default condition. Our data suggest that participants generally did not seek to share their good things with their entire Facebook network. Less than $21 \%$ of the good things from participants in the private default were posted to their newsfeed (and from only 6 of the 27 participants in this condition) and only an additional $23 \%$ were visible to friends viewing their $3 \mathrm{GT}$ profile. They left the remaining $56 \%$ as private. The public group made $14 \%$ of their items private and set $45 \%$ as visible to friends viewing their $3 \mathrm{GT}$ profile, but left only $40 \%$ in the default of being posted to their wall. Individual choices, however, varied greatly (Figure 5). 
Three of the participants we interviewed thought of what they recorded in 3GT as being primarily for themselves. When asked about sharing their good things with others, they raised concerns about not wanting to add to the "stuff" on Facebook or to "spam the rest of the world." For two, this meant rarely posting publicly and almost never posting to their newsfeed. For a third, this meant using different methods of posting depending on the content: "I'm more cognizant of cluttering other peoples' feeds than some of my friends. That's why I use different versions of the application."

Another participant wanted to share quite often with her friends, but was also very conscientious about over-posting. This meant occasionally setting posts to be private and often setting them not to appear on her newsfeed:

mostly when I make things private, it's more because I think they'd be boring or insignificant to my friends, not because they're actually things I wouldn't want my friends to know about. I just don't want to clog up their Facebook with it.... A lot of the people I'm friends with wince about having games and other non-status update things all over their pages. And so I don't want to get winced about.

One other participant thought of his posts as having an audience of his Facebook friends who were 3GT users. He was also concerned about over-posting to public spaces, but with his more social view of the application, this meant that he posted less frequently (less than once every three days), and only when an event met a "higher standard." He also noted that the online nature of the intervention prevented him from posting many good things that happened in his workplace, which he felt would have violated his non-disclosure agreement with his employer even if posted privately. This is one downside of moving the intervention online. This participant would also have preferred that a more restricted group of friends than his entire Facebook contact list be able to see items posted to 3GT, and for this reason never posted to his newsfeed. Even then, he would have preferred that the application require him to approve 3GT contacts separately from Facebook friends, so that friends signing up for the application would not automatically be able to view his good things list.

Desire for social interactions. Despite their reluctance to post too many good things publicly, five of the participants we interviewed were very positive about social interactions prompted by 3GT. One participant said that her status updates rarely received many comments, but when posting a good thing, "I got a TON of comments.... Lots of people said they liked that, and they responded to that and congratulated me... so yeah, people do definitely comment on them when they are created from Facebook and publicized [in newsfeeds]."

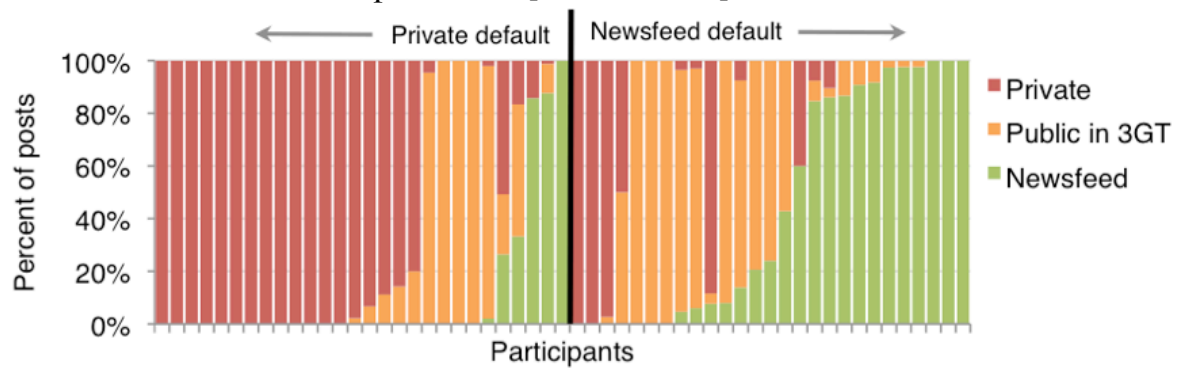

Fig. 5. Individual posting behavior and privacy defaults. Each column represents one active participant. 
Other participants were hopeful that 3GT use would prompt more social interactions than it had. According to one, "I could imagine a world where the good things entered in the system were used to start conversations..." Another felt frustrated by the lack of feedback on the good things she posted:

And it would have been cool to have somebody reacting to, like you said, the sort of social interaction over the content of the posts that I've done... To have some of these things - "oh, I see you posted something", or just some reaction. Because sometimes it feels like you're out there, putting stuff out in the world and you're not getting any feedback, you know?

One participant had posted "can't wait to see my friends' 3GT!" as one of his good things. During the interview he said that he liked looking at friends' good things because "it's like highlights of my friends' lives." He had followed up on some of the good things he saw his friends post, sometimes through Facebook and sometimes through other channels. Another reported, "it's just nice being able to see good things happening to other people." These participants are not alone in reading friends' profiles. Of the 4118 recorded page views, $16 \%$ were views of either an individual friend's 3GT profile (403 views) or a timeline view of all of their friends' 3GT posts (257 views). 25 of the 55 active participants viewed friends' good things using 3GT, and 24 used the application interface to invite friends.

Most participants wanted the 3GT application to make others' activity more salient. While we had assumed that people would post good things to their newsfeed if they wanted comments or discussion about good things, this was not true among participants we interviewed. They wanted more social interaction - such as the ability to comment on good things - within the application but not in their newsfeeds where it would be visible to friends not using 3GT. Representing a contrary view is one interview participant whose son also used 3GT. She said had never looked at his 3GT profile because she "figured it was private."

Effects of sharing. We had hypothesized that more public posts would attract the attention of a participant's friends, and that they would receive increased social support as a result, leading to both more posts per day and a greater retention rate. We did not find statistically significant differences in either of these activity measures. Our interviews also show that viewing others' activity in 3GT can sometimes lead to decreased activity. After beginning use of 3GT, one participant regularly posted good things three times per day. She later looked at friends' profiles and saw they were posting less frequently, and began to think that "maybe I was taking it too seriously, like a school assignment or something, if I didn't do it one day," and subsequently decreased how often she posted.

\subsection{Reminders}

Of the 55 active participants, 30 would receive system-generated reminders and 25 would not. Of those who completed the pre-test, 95 were assigned to the reminder condition and 95 were not, so there is no significant difference in retention rates at one week. Among the active users, people receiving reminders posted the same number of good things per day (0.82), on average, as those in the no-reminder group 
(0.83). The interviews with participants also helped us assess the effect of the reminders. While we had attempted to make the reminders "polite" and not too intrusive, we apparently erred in making the reminders too subtle. One participant did not know she had received reminders until we asked about them in the interview, and said that she needed something more prominent, like an email. Contrary to this, another participant liked that the reminders were integrated into Facebook: "I like the part that it sends me a note when I forget. I really like that! Having it remind me when I've forgotten is really cool."

User to User Reminders: Though many participants looked at each others' 3GT profiles, only seven participants sent a total of 14 reminders to their friends using the built-in reminder system. One participant we interviewed reacted strongly against the idea of reminding others to post using the interface. "No!... No, it's not my job." Another participant said that she enjoyed using 3GT more after she stopped thinking of it as "work" or something she had to do, and was concerned that pushing people to participate would cause them discomfort. She would be more inclined to prompt friends for participation if she knew they are "seriously combating depression, or it's something that they're really serious about and want to be doing, and they want me to help them get that done." Another participant felt more comfortable reminding her friends to post, but did not want to use the built in system for that: "I don't really like Nudge functions because they don't seem very personal. I'd rather go to their Wall and leave a sentence that was, you know, specifically from me."

\section{Design Implications}

The results of our study of the 3GT deployment highlight both strengths and weaknesses of the current 3GT design. Some aspects of 3GT offer positive examples for future intervention design, while others indicate particular design challenges for integrating wellness applications with social network sites.

Integrating wellness interventions into Facebook is a viable option. Our participants' dropout rates compare favorably to "open access" websites with as few as $1 \%$ active participants who return to the site after signing up [14]. Though this comparison is between different of wellness interventions, it lends support to the idea that Facebook-based interventions may assist adherence by facilitating easier access to the exercise materials.

Adding social features to an individual exercise can be beneficial, but not as a replacement to private participation. The social aspects of the intervention, i.e., sharing with others and viewing others' posts, were employed and deemed valuable by users. Specifically, people appreciated seeing others' posts and enjoyed receiving comments on their own. While previous studies identified privacy as barrier to integrating a wellness intervention with an existing social network site [13], we found that audience issues were a greater concern for 3GT participants. 3GT participants did not want all posts to be public, mostly out of concern of over-sharing. As one participant said, "I like the fact that I can choose whether things are private or public. Because if they were all public, some of them I wouldn't write, because I thought they 
weren't significant enough to sort of fill up others' Facebook pages with." A larger study would be required to determine effects on retention rate and clinical benefits.

Including intervention-specific user interface structure is beneficial. Compared with the relatively unstructured format of the two Three Good Things groups we analyzed, the addition of a separate, private field for participants to input the "reason" for each good thing encouraged them to complete the exercise as originally designed. While this appears obvious in hindsight, it is clear from the example of the groups that naïve or opportunistic implementations can miss critical details.

Employing built-in mechanisms for social interaction is not ideal. As mentioned, users employed and appreciated the social features of $3 \mathrm{GT}$, but few routinely shared their posts with members of their Facebook network beyond users of the 3GT application. There is some evidence that users would like to share their good things with people they know (i.e., existing members of their social networks as opposed to strangers in a dedicated "Three Good Things" group) but not with everyone they know. We believe that "friends of mine who are also using 3GT" would provide an effective first approximation for the users we interviewed, though many wanted more of their friends to actively use the application. Moreover, users were wary of cluttering their friends' news feeds, and would prefer to more publicly share only big events and accomplishments rather than everyday triumphs.

Subtle reminders are not effective for everyone. Using a "gentle" reminder mechanism (e.g. the Facebook notification interface) to remind participants to carry out the intervention activities was not found to be useful in this study. For the most part, participants did not appear to notice the reminders. We do not know if more assertive reminders would have increased participation (or, conversely, annoyed users enough to abandon the exercise).

\section{Conclusion}

Through our deployment and study of 3GT, we have found that it is possible to transfer wellness interventions to social networking sites and deliver benefits to users. There are indications that including a social component with a personal wellness exercise is desirable, but some care must be taken with how the social features are designed. In addition, we are in the process of collecting data regarding the impact of 3GT on participants' mood over time in order to compare the efficacy with the reported benefits of Seligman's offline individual exercise.

People are wary of polluting their friends' feeds with things that are too small or that do not fit the genre of what they usually share as updates. For some people who do want to share, their Facebook network is too broad of an audience and they wanted a way to share with a smaller, more controlled list of contacts. A structured reflection feature - the prompt to provide a reason - was beneficial. Notifications and reminders are needed and wanted, but they should not be so polite that they go unnoticed. Overall, integrating health and wellness applications into existing social network sites is indeed promising and should be explored further, with highest priority given to design of features that control the sharing of information with friends without eliminating it. 


\section{References}

1. Anhøj, J and Jensen, AH. (2004). "Using the Internet for life style changes in diet and physical activity: a feasibility study," J Med Internet Res 8;6(3):e28.

2. Blank, TO and Adams-Blodnieks, M. (2007). "The who and the what of usage of two cancer online communities," Computers in Human Behavior.

3. Consolvo, S et al. (2008). "Flowers or a Robot Army? Encouraging Awareness \& Activity with Personal, Mobile Displays," UbiComp 2008.

4. Grimes, A; Bednar, M; Bolter, JD; Grinter, RD. (2008.) "EatWell: Sharing NutritionRelated Memories in a Low-Income Community," CSCW 2008.

5. Klemm, P et al. (2003). "Online Cancer Support Groups: A Review of the Research Literature," CIN: Computers, Informatics, Nursing 21(3): 136-142.

6. Leslie, E; Marshall, AL; Owen, N; Bauman, A. (2005). "Engagement and retention of participants in a physical activity website," Prev Med 40(1):54-59.

7. Lin, JJ; Mamykina, L; Lindtner, S; Delajoux, G; Strub, HB. (2006) "Fish'n'Steps: Encouraging Physical Activity with an Interactive Computer Game," UbiComp 2006.

8. Mamykina, L; Mynatt, E; Davidson P; Greenblatt, D. (2008). "MAHI: investigation of social scaffolding for reflective thinking in diabetes management," CHI 2008.

9. Richardson, C; Brown, BB; Foley, S; Dial, KS; Lowery, JC. (2005). "Feasibility of Adding Enhanced Pedometer Feedback to Nutritional Counseling for Weight Loss," Med Internet Res 7(5): e56.

10. Tate, DF; Jackvony, EH; Wing, RR. (2003). "Effects of Internet behavioral counseling on weight loss in adults at risk for type 2 diabetes: a randomized trial," JAMA 289(14):18331836.

11. Positive Psychology Center. University of Pennsylvania. http://www.ppc.sas.upenn.edu/.

12. Wellman, B and Wortley S. 1990. "Different strokes from different folks: Community ties and social support," American Journal of Sociology: 558-588.

13. Olsen, E; Kraft, P. (2009). "ePsychology: A pilot study on how to enhance social support and adherence in digital interventions by characteristics from social networking sites," Persuasive 2009.

14. Facebook Press Page. http://www.facebook.com/press/info.php?statistics

15. Christensen, H; Griffiths, KM; Farrer L. (2009). "Adherence in Internet Interventions for Anxiety and Depression," J Med Internet Res 11(2): e13.

16. Wangberg, SC; Bergmo, TS and Johnsen, JAK. (2008). "Adherence in Internet-based interventions," Patient Preference and Adherence 2(2007): 1.

17. Ellison, NB; Steinfield, C; Lampe, C. (2007). "The benefits of Facebook 'friends:' Social capital and college students' use of online social network sites," Journal of ComputerMediated Communication, 12(4): I.

18. Consolvo, S; Everitt, K; Smith, I; Landay, J. (2006.) "Design requirements for technologies that encourage physical activity," CHI 2006.

19. Weinberg, $\mathbf{N}$ et al. (1996). "Online Help: Cancer Patients Participate in a ComputerMediated Support Group," Health \& Social Work 21(1): 24-29.

20. Welbourne, J; Blanchard, A; Boughton, MD. (2009). "Supportive communication, sense of virtual community and health outcomes in online infertility groups," Communities and Technologies 2009.

21. Khaled, R; Barr, P; Noble, J; Biddle; R. (2006). "Investigating Social Software as Persuasive Technology," Persuasive 2006. LNCS 3962: pp. 104-107. Springer.

22. Seligman, M; Steen, T; Park, N; Peterson, C. (2005). "Positive Psychology Progress," American Psychologist 60(5), 410-421. 